
Aus der Landwirtschantichen Universităt Wroclaw', der Justus-Liebig-Universitat Giessen ${ }^{2}$ und dem
Forschungsinstitut fur dic Biologie landwirtschaflicher Nutztiere (FBN), Dummerstorf

JERZY JUSZCZAK', GEORG ERHARDT T ${ }^{2}$, MARIAN KUCZAJ', RYSZARD ZIEMINSSII' und LOTHAR PANICKE ${ }^{3}$

\title{
Zusammenhang zwischen $\kappa$-Casein und $\beta$-Lactoglobulin-Varianten mit der Milchleistung und der Nutzungsdauer von Rindern der Rassen Schwarzbuntes Rind und Polnisches Rotvieh
}

\begin{abstract}
Summary
Title of the paper: Relations between genetic variants of $\kappa$-casein and $\beta$-lactoglobulin and performance of Black and White and Polish Red cattle

Performance of 184 cows from 4 herds of Black and White (SR) and 178 cows from 4 herds of Polish Red breed (RP) was compared due to genotype encoding milk proteins: $k$-casein and $\beta$-lactoglobulin. Frequence of inividual genotypes differend significantly both between herds within the breed. Statistically significant influence of genotype was found in relation to milk fat content in SR cattle $\left(\kappa-\mathrm{CN}^{\mu}>\kappa-\mathrm{CN}^{\mathrm{BB}}\right.$ and $\beta-\mathrm{LG}^{\mathrm{BB}}>\beta$. $\left.L G^{A B}>\beta-L G^{\Lambda \Lambda}\right)$ and in RP cattle milk protein yield $\left(\kappa-C N^{B B}>\kappa-C N^{\wedge \Lambda}\right)$ and fat content $\left(\beta-L G^{\wedge \wedge}>\beta-L G^{\wedge \theta}\right.$ and $\left.\beta-L G^{\mathrm{BB}}\right)$. The highest protein content and protein yield in milk was found in cows of both breeds carrying $\mathrm{k}$ $\mathrm{CN}^{\mathrm{BB}}$ genotype ( $\left.\mathrm{P}>0.05\right)$. Significant relation with the age of first calving was stated in $\mathrm{SR}$ cows: $\mathrm{K}-\mathrm{CN}^{\mathrm{AA}}>\mathrm{K}-$ $C N^{A B}$, in $R P$ cows $K-C N^{B B}>K-C N^{A B}$ and in SR cows $\beta-L G^{B B}>\beta-L G^{A B}$. Other traits such as longevity, life-time number of calvings and lactations, life-time yield were not found significantly related to searched genotypes although some trends were observed.
\end{abstract}

Key Words: cattle, Black and White, Polish Red Breed, $K$-casein, $\beta$-lactoglobulin, performance traits, longevity

\section{Zusammenfassung}

Die Milchleistung und die Nutzungsdauer wurden von 184 und 178 Kuhen in je 4 Schwarzbunt- (SR) und Polnischen Rotviehherden (RP) verglichen zwischen repräsentativen Genotypgruppen der Milchproteinfraktionen des $\mathrm{K}$-Casein $(\mathrm{K}$-CN) und des $\beta$-Lactoglobulin $(\beta-L G)$. Sowohl zwischen den Rassen als auch zwischen ćen Herden wurden wesentliche Differenzen der Frequenz von einzelnen Genotypgruppen festgestellt. Ein Zusammenhang zwischen dem Genotyp und der Milchleistung konnte nur bei SR fur den prozentualen Fettgehalt in der Milch $\left(\kappa-C N^{\Lambda \Lambda}>K-C^{8 B}\right.$ und $\beta-L G^{\mathrm{BB}}>\beta-L G^{\Lambda \mathrm{B}}>\beta-L G^{\wedge \Lambda}$ ) sowie bei $R P$ flir dic Proteinleistung $\left(K-\mathrm{CN}^{\mathrm{BB}}>\kappa-\mathrm{CN}^{\mathrm{AA}}\right)$ und den Fettgebalt $\left(\beta-\mathrm{LG}^{\mathrm{AA}}>\beta-\mathrm{LG}^{\mathrm{BB}}>\beta-\mathrm{LG}^{\mathrm{AB}}\right)$ ermittelt werden. Weiterhin wurden signifikante Relationen zum Erstkalbealter bei SR fur $\mathrm{K}-\mathrm{CN}^{\wedge \wedge}>k-\mathrm{CN}^{\wedge \mathrm{B}}$ und fur $\beta-\mathrm{LG}^{\mathrm{BB}}>\beta$ $\mathrm{LG}^{\mathrm{AB}}$ sowie bei RP für $\mathrm{K}-\mathrm{CN}^{\mathrm{BH}}>\mathrm{K}-\mathrm{CN}^{\lambda \mathrm{B}}$ festgestellt.

Schllisselwörter: Milchrind, Schwarzbuntes Rind, Polnisches Rotvieh, $\kappa$-Caseins, $\beta$-Lacioglobulin, Nutzungsdauer

\section{Einleitung}

Das Interesse für Milchprotein und seine polymorphen Varianten ist im Zusammenhang mit Verarbeitungseigenschaften der Milch begrïndet. Eine wichtige Rolle wird dabei dem $\mathrm{k}$-Casein zugesprochen. Obwohl es nur 7 - $8 \%$ des Gesamtcaseins in der Milch ausmacht, soll seine genetische Variante BB eine verbesserte Käsereitauglichkeit verursachen. Diese Milch charakterisiert sich mit einer verkürzten Gerinnungszeit 
JUSZCZAK u,a.: $k$-Casein und $\beta$-Lactoglobulin-Varianten bei Rindern der Rassen Schwarzbuntes Rind und Polnisches Rotvieh

und einer besseren Gallertefestigkeit (AALLTONEN und ANTILA, 1987; SCHULTECOERNE und PABST, 1991). Als Ursache dafür werden der höhere $\kappa$-Casein- und der höhere Caseingehalt in $\kappa$-Casein BB-Milchen sowie kleinere Micellengrösse angenommen. Ausserdem erhöht sich die Käsereiausbeute (MARZIALI und NG-KWAIHANG, 1986). Das zweite untersuchte Milchprotein ist $\beta$-Lactoglobulin. Es spielt eine andere Rolle als Enzym bei metabolischen Prozessen in den milcherzeugenden Alveolen. Es tritt in genetisch bedingten polymorphen Formen auf. Während AALLTONEN und ANTILA (1987), FELENCZAK et al. (1983), MARIANI und LEONI (1985), NG-KWAI-HANG et al. (1986) eine Überlegenheit bei Kühen mit einem $\beta$-Lactoglobulin AA Genotyp gegenüber BB Genotyp ausweisen, beobachten ALEANDRI et al. (1997) in Abhängigkeit von der zu produzierenden Käsesorte unterschiedliche Einflüsse der beiden Genorte $\kappa$-Casein und $\beta$-Lactoglobulin.

Zusammenhänge zwischen Milchproteingenotypen und quantitativen Eigenschaften waren Gegenstand zahlreicher Untersuchungen in der Vergangenheit. Dabei zeigt sich, dass Kühe mit einem hohen Proteingehalt entweder die $\kappa$-Casein-Variante $B$, die $\beta$ Lactoglobulin-Variante A oder $\alpha_{S I}$ - Casein C tragen (ERHARDT, 2000). Die Ergebnisse sind nicht immer gleich, was mit dem Einfluss der Genotyp-Umwelt-Interaktionen sowie der Genexpression erklärt werden könnte. Überwiegend findet man die Ansicht, dass die für technologische Milcheigenschaften positiven Genotypen $\kappa$-Casein BB und $\beta$-Lactoglobulin AA über eine Überlegenheit nur bei den Milchinhaltsstoffen verfügen, während sie in Milchleistung, Fett- und Proteinertrag unterlegen sind (SOWINSKI, I993; PANICKE et al., 1996, 1997; TRAKOVICKA et al., 1997). Genetische Studien zeigen, dass die Caseine als Cluster organisiert sind (FERRETTI et al., 1990).

Es wird in Regionen mit entwickelter Käseproduktion darüber nachgedacht, bei der Selektion der Milchrinder den $\kappa$-Casein Genotyp zu berücksichtigen (MARIANI und PECORARI, 1987; SCHAAR, 1986; SOWINSKI, 1993). In Bullenkatalogen u.a. in USA, Holland und in der Schweiz, befinden sich aus diesem Grund neben den Informationen über die Zuchtwerte zusätzlich Informationen über den Genotyp. Dieses Problem muss jedoch auch weiterhin sowohl im Zusammenhang mit Parametern der Nutzungsdauer als auch der Fruchtbarkeit (PANICKE et al., 1998) und Vitalität von Kälbern (GRAML et al., 1988; WALAWSKI et al,, 1997) geprüft werden.

Mit diesen Untersuchungen soll ein Beitrag zur Milchleistung und Nutzungsdauer in den Genotypvarianten des $\kappa$-Caseins $(\kappa-\mathrm{CN})$ und des $\beta$-Lactoglobulins $(\beta-\mathrm{LG})$ von Polnischen Schwarzbunten (SR) und Polnischem Rotvieh (RP) bei unterschiedlichem Leistungsniveau erbracht werden.

\section{2.} Material und Methoden

Die Untersuchungen der Milchproteinpolymorphismen wurden an 362 Jungkühen in der ersten Laktation in vier Schwarzbunt-Herden (SR) ohne oder mit geringem Holstein-Friesian Anteil (bis zu 12,5\%) und in vier Herden mit Polnischem Rotvieh (RP) in Polen durchgeführt (Tab. 1).

Die weitere Prüfung erstreckt sich über die gesamte Nutzungsdauer bis zum Abgang der Kühe. Von allen 184 SR Kühen und 178 PR Kühen wurden Milchproben mit 
Tabelle 1

$\frac{\text { Versuchsumfang in den Schwarzbunt und Rotyieh-Herden (Structure of the analysed material) }}{\text { Rasse }}$

\begin{tabular}{|c|c|c|c|c|c|}
\hline Rasse & 1 & 2 & 3 & 4 & Gesamt \\
\hline $\begin{array}{l}\text { Schwarzbunte ( SR) } \\
\text { Poln. Rotvieh ( PR) }\end{array}$ & $\begin{array}{l}62 \\
41\end{array}$ & $\begin{array}{l}64 \\
72\end{array}$ & $\begin{array}{l}14 \\
29\end{array}$ & $\begin{array}{l}44 \\
36\end{array}$ & $\begin{array}{l}184 \\
178 \\
\end{array}$ \\
\hline
\end{tabular}

Natriumazid konserviert. Die Milchproben wurden im Labor des Institutes für Haustiergenetik der Justus-Liebig-Universität Giessen hinsichtlich der genetischen Varianten im $\alpha_{S_{1}}$-Casein, $\alpha_{S_{2}}$-Casein, $\beta$-Casein, $\alpha$-Casein, $\alpha$-Lactalbumin sowie $\beta$ Lactoglobulin durch isoelektrische Fokussierung unter Verwendung freier Trägerampholyte in ultradünnen Polyacrylamidgelen (SEIBERT et al., 1985; ERHARDT, 1989, 1993) typisiert. Die Milchleistung der Kühe wurde aus den Ergebnissen der amtlichen Milchleistungskontrolle bereitgestellt. Die weiteren erforderlichen Informationen zur Nutzungsdauer wurden aus der zootechnischen Dokumentation übernommen. In diese Auswertung wurden die Kühe der repräsentativen Genotypen der kCasein- und $\beta$-Lactoglobulinfraktionen mit folgenden Merkmalen einbezogen:

Milchmenge $(\mathrm{kg})$, Fettmenge $(\mathrm{kg})$ und Eiweissmenge $(\mathrm{kg})$ sowie der prozentuale Fettund Eiweissgehalt der Erstlaktationsperiode von 305 Tagen, Alter der ersten Kalbung, Lebensdauer, Anzahl der Laktationen im Leben und die Lebensleistung.

Die Jungkuhleistungen in der ersten Laktation wurden korrigiert auf die systematischen Einflussfaktoren Kalbezeitpunkt (HYS) und Kalbealter (EKA) innerhalb jeder Rasse nach ŻUK et al. (1980). Der Effektschätzung dafür liegt das allgemeine Modell:

zugrunde. Darin bedeuten:

$$
y_{i j k}=\mu+H Y S_{j}+E K A_{k}+\varepsilon_{i j k}
$$

$$
\begin{aligned}
& \mathrm{y}_{\mathrm{ijk}}=\text { Beobachtungswert der 305-Tageleistung der i-ten Kuh } \\
& \mu \quad=\text { allgemeines Mittel } \\
& \text { HYS = fixer Einfluss von Herde, Jahr und Saison im j-ten Abkalbezeitpunkt } \\
& \mathrm{EKA}=\text { fixer Einfluss des k-ten Erstkalbealters } \\
& \varepsilon_{\mathrm{ijk}} \text {. }=\text { Resteffekt }
\end{aligned}
$$

Im Rechenzentrum des Lehrstuhls für Rinderzucht und Milchproduktion der Landwirtschaftlichen Universität Wroclaw wurde das Material statistisch bearbeitet. Für jedes Merkmal wurden innerhalb genetischer Gruppen der Kühe die Mittelwerte und die Standardabweichungen ermittelt und mit dem Duncan-Test verglichen.

3. Ergebnisse

Aufgrund der durchgeführten Typisierung der polymorphen Genotypen von $\kappa$-Casein und $\beta$-Lactoglobulin, wurde eine Analyse der genetischen Struktur in den Herden erstellt (Tab, 2 und 3). Daraus geht hervor, dass sich die beiden untersuchten Rassen in der Genotypen- und Allelfrequenz beider Proteinfraktionen unterscheiden. Beim SR (Tab. 2) tritt die $\kappa$-Casein-Fraktion ( $\kappa-C N$ ) mit den Allelen $A, B$ und $E$ in fünf Genotypenvarianten $\mathrm{AA}, \mathrm{AB}, \mathrm{BB}, \mathrm{AE}$ und $\mathrm{BE}$ auf. Die Relation zwischen dem $\mathrm{A}$ - und $\mathrm{B}$ Allel liegt mit 78 zu $21 \%$ etwa bei 4:1. Die $\kappa-C N$ Genotypvarianten AE und BE wurden nur in einzelnen Fällen nachgewiesen. Die Frequenz des E-Allels in der Po- 
pulation betrug im Mittel unter $1 \%$ und erreichte in keiner Herde $2 \%$. Es wurde eine Differenzierung der Allelfrequenz zwischen den Herden beobachtet. Sie variiert im Mittel der Herden für das $\kappa$-Caseinallel A zwischen $83 \%$ in Herde 1 und $71 \%$ in Herde 3. Dies ist für den heterozygoten $\kappa$-Caseingenotyp $A B$ mit einem differenzierten Anteil von $26 \%$ in Herde 1 und $43 \%$ in Herde 3 verbunden.

Die $\beta$-Lactoglobulin-Fraktion ( $\beta$-LG) bei den SR-Kühen weist für beide Allele $A$ und B etwa gleiche Anteile um $50 \%$ aus, Daraus folgt die zu erwartende Normalverteilung $\operatorname{der} \beta$-LG-Genotypen AA mit 27\%, AB mit $46 \%$ und BB mit $27 \%$. Ähnlich wie beim $\kappa-\mathrm{CN}$ treten wesentliche Differenzierungen in der Allelfrequenz mit etwa $60 \%$ und 40 $\%$ zwischen den Herden 3 und 4 auf. Der höchste Anteil des heterozygoten $\beta$-LG-Genotyps $\mathrm{AB}$ ist bei Gleichverteilung der beiden Allele in Herde 1 zu erwarten.

\section{Tabelle 2}

Milchproteingenotypen und Allelfrequenz von $\kappa$-Casein und $\beta$-Lactoglobulin bei Schwarzbunten (SR) (Frequencies of genotypes and polimorphic genes of $\kappa$-casein and $\beta$-lactoglobulin fractions in cows of Blackand-White breed)

\begin{tabular}{|c|c|c|c|c|c|c|c|c|c|}
\hline \multirow[t]{2}{*}{ Herde } & \multirow{2}{*}{ Anzahl Kuine } & \multicolumn{5}{|c|}{ Genotypvarianten } & \multicolumn{3}{|c|}{ Allelfrequenz } \\
\hline & & AA & $A B$ & $\mathrm{BB}$ & $\mathrm{AE}$ & $\mathrm{BE}$ & A & $\mathrm{B}$ & E \\
\hline \multicolumn{10}{|c|}{$\kappa$-Casein } \\
\hline 1 & 62 & 0,678 & 0,258 & 0,032 & 0,032 & & 0,832 & 0,161 & 0,016 \\
\hline 2 & 64 & 0,609 & 0,344 & 0,047 & & & 0,781 & 0,219 & \\
\hline 3 & 14 & 0,500 & 0,429 & 0,071 & & & 0,714 & 0,286 & \\
\hline 4 & 44 & 0,568 & 0,341 & 0,068 & & 0,023 & 0,739 & 0,250 & 0,011 \\
\hline Gesamt & 184 & 0,614 & 0,321 & 0,049 & 0,011 & 0,005 & 0,778 & 0,213 & 0,008 \\
\hline \multicolumn{10}{|c|}{$\beta$-Lactoglobulin } \\
\hline 1 & 62 & 0,242 & 0,532 & 0,226 & & & 0,508 & 0,492 & \\
\hline 2 & 64 & 0,344 & 0,422 & 0,234 & & & 0,555 & 0,445 & \\
\hline 3 & 14 & 0,429 & 0,357 & 0,214 & & & 0,607 & 0,393 & \\
\hline 4 & 44 & 0,159 & 0,455 & 0,386 & & & 0,386 & 0,614 & \\
\hline Gesamt & 184 & 0,272 & 0,462 & 0,266 & & & 0,503 & 0,497 & \\
\hline
\end{tabular}

Beim Polnischen Rotvieh (RP) (Tab. 3) treten im untersuchten Bestand in der Proteinfraktion des $\kappa-\mathrm{CN}$ nur die zwei Allele A und B mit den drei Genotypen: AA, $\mathrm{AB}$ und $\mathrm{BB}$ auf. Das Allel $\mathrm{E}$ ist in keiner der untersuchten Herden zu finden. Dafür kommt das C-Allel beim B-LG im Anteil von 1-2\% hinzu.

Im Vergleich zum SR- wird in der gepruften Kühepopulation des RP eine geringere $\kappa$ Caseinallelfrequenz für A im Mittel mit $68 \%$ bei einer Variation von $60 \%$ in Herde 4 bis $74 \%$ in Herde 2 beobachtet. Die Relation zwischen dem $\kappa$-Caseinallel A und B verändert sich von etwa 80 : 20 bei SR auf $70 ３ 0$ bei RP respektive. Beim $\beta$-LG verändert sich diese Relation zwischen den Allelen $A$ und $B$ von $50: 50$ beim SR auf 22 : 74 beim RP mit einer Variationsbreite für das $\beta$-LG-Allel A im Mittel der Herden von $7 \%$ in Herde 1 bis $43 \%$ in Herde 4 entsprechend für das $\beta$-LG-Allel B von $57 \%$ in Herde 4 bis $92 \%$ in Herde 1 .

Die Diagramme in den Abbildungen 1 und 2 illustrieren am besten die grosse Differenzierung der genetischen Struktur der gepruften Herden. Dabei muss man betonen, dass die dargestellte genetische Struktur der geprilften Herden an völlig zufallsmässig ausgewählten Tieren untersucht wurde. 
Tabelle 3

Milchproteingenotypen und Allelfrequenz von $\kappa$-Casein und $\beta$-Lactoglobulin bei Polnischem Rotvieh (RP) Frequencies of genotypes and polimorphic genes of $\kappa$-casein and $\beta$-lactoglobulin fractions in cows of Polish Red
breed)

\begin{tabular}{|c|c|c|c|c|c|c|c|c|c|}
\hline \multirow[t]{2}{*}{ Herde } & \multirow[t]{2}{*}{ Anzahl Kuhe } & \multicolumn{5}{|c|}{ Genotypvarianten } & \multicolumn{3}{|c|}{ Allelfrequenz } \\
\hline & & AA & $\mathrm{AB}$ & $\mathrm{BB}$ & $\mathrm{AC}$ & $\mathrm{BC}$ & A & $\mathrm{B}$ & C \\
\hline \multicolumn{10}{|c|}{$\kappa$-Casein } \\
\hline 1 & 41 & 0,366 & 0,512 & 0,122 & & & 0,622 & 0,378 & \\
\hline 2 & 72 & 0,514 & 0,458 & 0,028 & & & 0,743 & 0,257 & \\
\hline 3 & 29 & 0,483 & 0,448 & 0,069 & & & 0,707 & 0,293 & \\
\hline 4 & 36 & 0,306 & 0,583 & 0,111 & & & 0,597 & 0,403 & \\
\hline Gesamt & 178 & 0,433 & 0,494 & 0,073 & & & 0,680 & 0,320 & \\
\hline \multicolumn{10}{|c|}{$\beta$-Lactoglobulin } \\
\hline $\mathrm{I}$ & 41 & & 0,146 & 0,829 & & 0,025 & 0,073 & 0,915 & 0,012 \\
\hline 2 & 72 & 0,041 & 0,278 & 0,528 & 0,028 & 0,125 & 0,194 & 0,729 & 0,077 \\
\hline 3 & 29 & 0,069 & 0,241 & 0,586 & 0,035 & 0,069 & 0,207 & 0,741 & 0,052 \\
\hline 4 & 36 & 0,166 & 0,528 & 0,306 & & & 0,431 & 0,569 & \\
\hline Gesamt & 178 & 0,062 & 0,292 & 0,562 & 0,017 & 0,067 & 0,216 & 0,742 & 0,042 \\
\hline
\end{tabular}

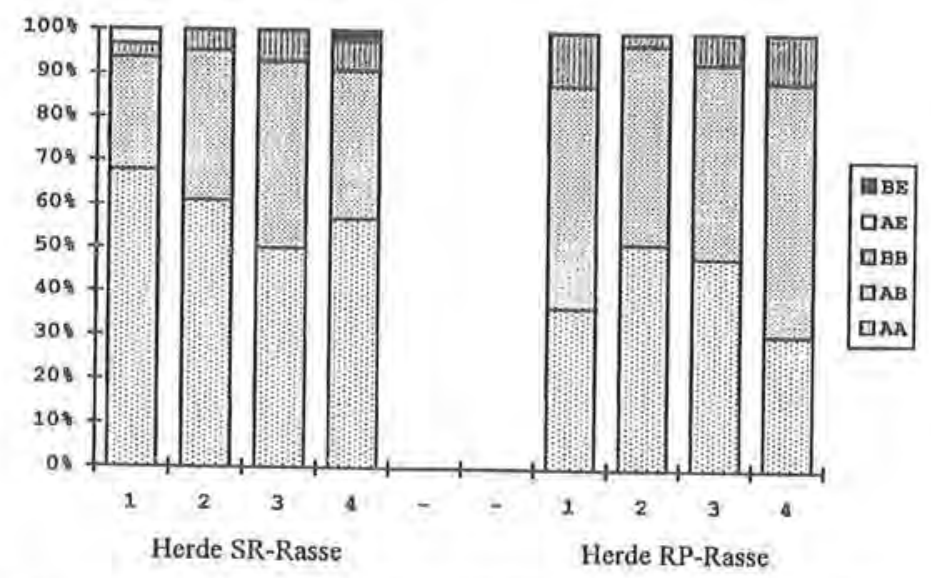
Abb. I; Frequenz der $\mathrm{k}$-Caseingenotypen in einzelnen Herden (Frequencies of $\mathrm{K}$-casein genotypes in cattlc
herds)

Die Milchleistungen von Kühen beider Rassen SR (Tab. 4) und RP (Tab. 5) in der ersten 305-Tage-Laktation sind unter Berücksichtigung des $\kappa$-Casein- und $\beta$ Lactoglobulin-Genotyps als Absolutleistung sowie korrigiert nach Kalbezeitpunkt und Erstkalbealter dargestellt. Bei dem SR treten statistisch gesicherte Differenzen zwischen den Milchproteinvarianten nur für den prozentualen Fettgehalt auf $\kappa-\mathrm{CN}^{\lambda \wedge}$ $>\mathrm{K}-\mathrm{CN}^{\mathrm{BB}}$ sowie $\beta-\mathrm{LG}^{\mathrm{BB}}>\beta-\mathrm{LG}^{\mathrm{AA}}$ und $\beta-\mathrm{L} \mathrm{G}^{\mathrm{AB}}$. Die Unterschiede zwischen den $\mathrm{K}-\mathrm{CN}$ Genotypen $\mathrm{AA}$ und $\mathrm{BB}$ um $13 \mathrm{~kg}$ Fett und $4 \mathrm{~kg}$ Protein konnten ebenso wie die Unterschiede zwischen den $\beta$-LG-Genotypen AA und BB um $400 \mathrm{~kg}$ Milch, $8 \mathrm{~kg}$ Fett und $10 \mathrm{~kg}$ Protein nicht gesichert werden. Die um $400 \mathrm{~kg}$ höhere Milchleistung korrespondiert mit den geringsten Milchinhaltsstoffen von 3,96\% Fett und 3,18\% Protein. 


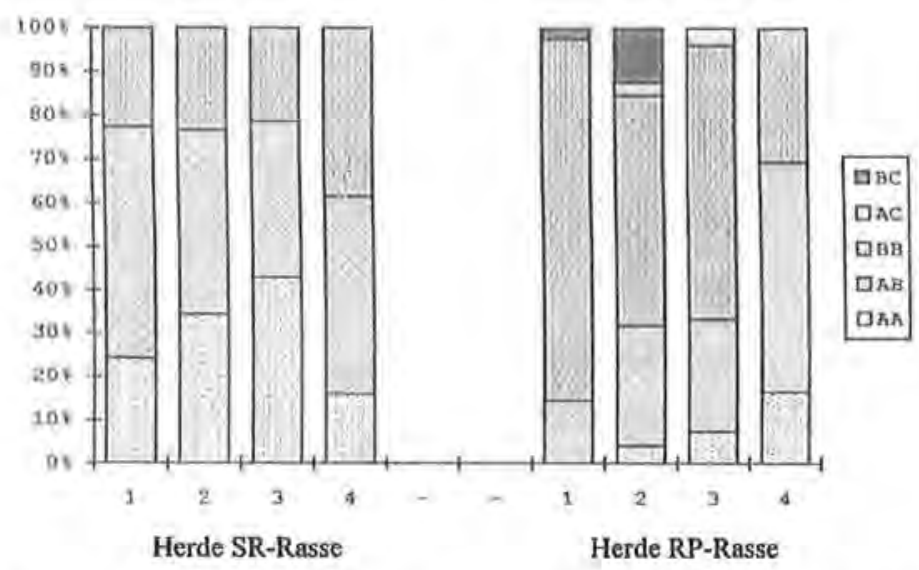

Abb. 2: Frequenz der $\beta$-Lactoglobulingenotypen in einzelnen Herden (Frequencies of $\beta$-lactoglobulin genotypes in cattle herds)

\section{Tabelle 4}

Milchleistung bei Schwarzbunten nach $\kappa$-Casein- und $\beta$-Lactoglobulin-Genotypvarianten (Milk performance in Black-and White breed due to $\kappa$-casein and $\beta$-lactoglobulin genotyps)

\begin{tabular}{|c|c|c|c|c|c|c|c|c|c|c|}
\hline \multirow[b]{3}{*}{$\begin{array}{c}\text { Geno- } \\
\text { typ }\end{array}$} & \multirow[b]{3}{*}{$\begin{array}{c}\text { Anzahl } \\
\text { Kühe }\end{array}$} & \multirow[b]{3}{*}{$\begin{array}{c}\text { Sym- } \\
\text { bol }\end{array}$} & \multicolumn{8}{|c|}{ Milchleistung der Jungkuhe in der 1. Laktation } \\
\hline & & & \multicolumn{4}{|c|}{ Absolutleistung } & \multicolumn{3}{|c|}{ Korrigierte Leistung } & \multirow[b]{2}{*}{$\begin{array}{c}\text { Prot.- } \\
\%\end{array}$} \\
\hline & & & $\begin{array}{l}\text { Milch- } \\
\text { kg }\end{array}$ & $\begin{array}{c}\text { Fett- } \\
\text { kg }\end{array}$ & $\begin{array}{l}\text { Prot.- } \\
\text { kg }\end{array}$ & $\begin{array}{l}\text { Milch- } \\
\text { kg }\end{array}$ & $\begin{array}{l}\text { Fett- } \\
\text { kg }\end{array}$ & $\begin{array}{c}\text { Prot.- } \\
\text { kg }\end{array}$ & $\begin{array}{c}\text { Fett- } \\
\%\end{array}$ & \\
\hline \multicolumn{11}{|c|}{ K-Casein } \\
\hline AA & 91 & $\begin{array}{l}X \\
s\end{array}$ & $\begin{array}{l}5009 \\
955\end{array}$ & $\begin{array}{c}203,4 \\
41,5\end{array}$ & $\begin{array}{c}160,0 \\
30,6\end{array}$ & $\begin{array}{l}5362 \\
1035\end{array}$ & $\begin{array}{c}217,7 \\
44,5\end{array}$ & $\begin{array}{c}171,5 \\
33,8\end{array}$ & $\begin{array}{c}4,06^{6} \\
0,30\end{array}$ & $\begin{array}{l}3,20 \\
0,14\end{array}$ \\
\hline $\mathrm{AB}$ & 47 & $\begin{array}{l}\bar{x} \\
s\end{array}$ & $\begin{array}{c}4796 \\
788\end{array}$ & $\begin{array}{c}190,0 \\
33,1\end{array}$ & $\begin{array}{c}154,2 \\
26,8\end{array}$ & $\begin{array}{c}5187 \\
830\end{array}$ & $\begin{array}{c}205,4 \\
34,7\end{array}$ & $\begin{array}{c}166,7 \\
28,4\end{array}$ & $\begin{array}{l}3,96 \\
0,24\end{array}$ & $\begin{array}{l}3,21 \\
0,16\end{array}$ \\
\hline BB & 8 & $\begin{array}{l}\bar{x} \\
\mathrm{~s}\end{array}$ & $\begin{array}{c}4850 \\
774\end{array}$ & $\begin{array}{c}184,0 \\
23,2\end{array}$ & $\begin{array}{r}158,1 \\
24,9\end{array}$ & $\begin{array}{c}5391 \\
845\end{array}$ & $\begin{array}{c}204,7 \\
26,8\end{array}$ & $\begin{array}{c}175,6 \\
26,2\end{array}$ & $\begin{array}{c}3,82^{\star} \\
0,26\end{array}$ & $\begin{array}{l}3,26 \\
0,19\end{array}$ \\
\hline \multicolumn{11}{|c|}{$\beta$-Lactoglobulin } \\
\hline AA & 37 & $\begin{array}{l}X \\
s\end{array}$ & $\begin{array}{l}5196 \\
893\end{array}$ & $\begin{array}{l}205,5 \\
37,1\end{array}$ & $\begin{array}{l}165,1 \\
29,3\end{array}$ & $\begin{array}{l}5547 \\
957\end{array}$ & $\begin{array}{l}219,2 \\
39,1\end{array}$ & $\begin{array}{c}176,3 \\
31,7\end{array}$ & $\begin{array}{c}3,96^{b} \\
0,27\end{array}$ & $\begin{array}{l}3,18 \\
0,16\end{array}$ \\
\hline $\mathrm{AB}$ & 68 & $\begin{array}{l}\bar{x} \\
s\end{array}$ & $\begin{array}{c}4891 \\
910\end{array}$ & $\begin{array}{c}195,2 \\
39,8\end{array}$ & $\begin{array}{c}157,1 \\
30,6\end{array}$ & $\begin{array}{c}5294 \\
935\end{array}$ & $\begin{array}{c}211,2 \\
40,9\end{array}$ & $\begin{array}{c}170,2 \\
31,8\end{array}$ & $\begin{array}{c}3,99^{c} \\
0,28\end{array}$ & $\begin{array}{l}3,21 \\
0,14\end{array}$ \\
\hline BB & 41 & $\begin{array}{l}\bar{x} \\
s\end{array}$ & $\begin{array}{c}4795 \\
860\end{array}$ & $\begin{array}{c}196,6 \\
37,7\end{array}$ & $\begin{array}{c}154,3 \\
26,3\end{array}$ & $\begin{array}{l}5150 \\
1011\end{array}$ & $\begin{array}{c}211,2 \\
43,3\end{array}$ & $\begin{array}{c}166,0 \\
32,2\end{array}$ & $\begin{array}{c}4,11^{\text {be }} \\
0,32\end{array}$ & $\begin{array}{l}3,23 \\
0,14\end{array}$ \\
\hline
\end{tabular}

- Korrektur auf Kalbealter und Kalbezeitpunkt Es bedeuten : ${ }^{a, c}$ - Signifikanz $(\mathrm{P}=5 \%)$

In der Stichprobe der Population des RP (Tab. 5) konnten Differenzen von $14 \mathrm{~kg}$ in der Proteinleistung aufgezeigt werden $\kappa-\mathrm{CN}^{\mathrm{BB}}>\kappa-\mathrm{CN}^{\mathrm{AA}}$, während Unterschiede um $400 \mathrm{~kg}$ Milch und $18 \mathrm{~kg}$ Fett zwischen den gleichen Genotypen ungesichert blieben. Zwischen den $\beta$-LG-Genotypen treten gesicherte Differenzen im prozentualen Fettgehalt auf $\beta-\mathrm{LG}^{\mathrm{AA}}>\beta-\mathrm{LG}^{\mathrm{BB}}$ und $\beta-\mathrm{LG}^{\mathrm{AB}}$.

Für die Analyse der Nutzungsdauer nach dem $\kappa-C N$ - und dem $\beta$-LG-Genotyp von Kühen beider Stichproben im ganzen Leben fanden die Parameter Erstkalbealter, Zwischenkalbezeit, Lebensdauer und Anzahl der Laktationen im Leben für SR (Tab. 
Tabelle 5

Milchleistung beim Polnischen Rotvieh nach $\mathrm{K}$-Casein und $\beta$-Lactoglobulin-Genotypvarianten (Milk performance in Polish Red breed due to $\kappa$-casein und $\beta$-lactoglobulin genotyps)

\begin{tabular}{|c|c|c|c|c|c|c|c|c|c|c|}
\hline \multirow[b]{3}{*}{$\begin{array}{c}\text { Geno- } \\
\text { typ }\end{array}$} & \multirow[b]{3}{*}{$\begin{array}{l}\text { Anzahl } \\
\text { Kühe }\end{array}$} & \multirow[b]{3}{*}{ Symbol } & \multicolumn{8}{|c|}{ Milchleistung der Jungkilhe in der 1. Laktation } \\
\hline & & & \multicolumn{3}{|c|}{ Absolutleistung } & \multicolumn{4}{|c|}{ Korrigierte Leistung } & \multirow[b]{2}{*}{$\begin{array}{c}\text { Prot- } \\
\%\end{array}$} \\
\hline & & & $\begin{array}{c}\text { Milch- } \\
\text { kg }\end{array}$ & $\begin{array}{c}\text { Fett- } \\
\text { kg }\end{array}$ & $\begin{array}{c}\text { Prot- } \\
\text { kg }\end{array}$ & $\begin{array}{c}\text { Milch- } \\
\text { kg }\end{array}$ & $\begin{array}{c}\text { Fett- } \\
\text { kg }\end{array}$ & $\begin{array}{c}\text { Prot.- } \\
\mathrm{kg}\end{array}$ & $\begin{array}{c}\text { Fett- } \\
\%\end{array}$ & \\
\hline \multicolumn{11}{|c|}{ k-Casein } \\
\hline $\mathrm{A \Lambda}$ & 60 & $\bar{x}$ & $\begin{array}{c}2578 \\
598\end{array}$ & $\begin{array}{c}109,9 \\
24,8\end{array}$ & $\begin{array}{c}80,9^{2} \\
17,6\end{array}$ & $\begin{array}{c}2733 \\
607\end{array}$ & $\begin{array}{c}116,4 \\
25,3\end{array}$ & $\begin{array}{c}85,8^{\circ} \\
17,9\end{array}$ & $\begin{array}{l}4,28 \\
0,33\end{array}$ & $\begin{array}{l}3,15 \\
0,17\end{array}$ \\
\hline $\mathrm{AB}$ & 54 & $\begin{array}{l}\bar{x} \\
\text { s }\end{array}$ & $\begin{array}{c}2587 \\
555\end{array}$ & $\begin{array}{c}110,8 \\
25,0\end{array}$ & $\begin{array}{l}82,7 \\
17,2\end{array}$ & $\begin{array}{c}2848 \\
619\end{array}$ & $\begin{array}{c}121,9 \\
27,6\end{array}$ & $\begin{array}{l}91,0 \\
19,5\end{array}$ & $\begin{array}{l}4,28 \\
0,35\end{array}$ & $\begin{array}{l}3,20 \\
0,18\end{array}$ \\
\hline BB & 10 & $\begin{array}{l}\bar{x} \\
\mathrm{~s}\end{array}$ & $\begin{array}{c}2927 \\
534 \\
\end{array}$ & $\begin{array}{c}126,9 \\
24,0 \\
\end{array}$ & $\begin{array}{c}94,1^{b} \\
18,9 \\
\end{array}$ & $\begin{array}{c}3103 \\
465 \\
\end{array}$ & $\begin{array}{c}134,8 \\
21,9 \\
\end{array}$ & $\begin{array}{c}99,7^{b} \\
16,9\end{array}$ & $\begin{array}{l}4,36 \\
0,35\end{array}$ & $\begin{array}{l}3,21 \\
0,19 \\
\end{array}$ \\
\hline \multicolumn{11}{|c|}{ B-Lactoglobulin } \\
\hline AA & 7 & $\begin{array}{l}\bar{x} \\
s\end{array}$ & $\begin{array}{c}2387 \\
355\end{array}$ & $\begin{array}{c}112,1 \\
23,3\end{array}$ & $\begin{array}{l}78,7 \\
17,7\end{array}$ & $\begin{array}{c}2566 \\
402\end{array}$ & $\begin{array}{l}120,7 \\
25,8\end{array}$ & $\begin{array}{l}84,5 \\
19,0\end{array}$ & $\begin{array}{c}4,67^{26} \\
0,29\end{array}$ & $\begin{array}{l}3,27 \\
0,25\end{array}$ \\
\hline $\mathrm{AB}$ & 27 & $\begin{array}{l}\bar{x} \\
s\end{array}$ & $\begin{array}{c}2649 \\
565\end{array}$ & $\begin{array}{l}112,1 \\
26,5\end{array}$ & $\begin{array}{l}84,8 \\
16,5\end{array}$ & $\begin{array}{c}2863 \\
563\end{array}$ & $\begin{array}{l}121,0 \\
26,1\end{array}$ & $\begin{array}{l}91,8 \\
16,9\end{array}$ & $\begin{array}{c}4,22^{\circ} \\
0,27\end{array}$ & $\begin{array}{l}3,22 \\
0,14\end{array}$ \\
\hline BB & 91 & $\begin{array}{l}\bar{x} \\
s\end{array}$ & $\begin{array}{c}2614 \\
593\end{array}$ & $\begin{array}{l}111,3 \\
25,0\end{array}$ & $\begin{array}{l}82,4 \\
18,1\end{array}$ & $\begin{array}{c}2814 \\
629\end{array}$ & $\begin{array}{c}119,8 \\
26,7\end{array}$ & $\begin{array}{l}88,6 \\
19,4\end{array}$ & $\begin{array}{c}4,27^{b} \\
0,34\end{array}$ & $\begin{array}{l}3,16 \\
0,18\end{array}$ \\
\hline
\end{tabular}

6) und RP (Tab. 7) Verwendung. Es gibt nur einen gesicherten Zusammenhang zum Erstkalbealter für den Genotyp $\kappa-\mathrm{CN}^{\mathrm{AA}}>\kappa-\mathrm{CN}^{\mathrm{AB}}$ und für den Genotyp $\beta-\mathrm{LG}^{\mathrm{BB}}>\beta$ $\mathrm{LG}^{\mathrm{AB}}$ bei SR und für den Genotyp $\kappa-\mathrm{CN}^{\mathrm{BB}}>\kappa-\mathrm{CN}^{\mathrm{AB}}$ beim $\mathrm{RP}$ entsprechend mit 22 , 27 und 50 Tagen. In der weiteren Nutzung nehmen zootechnische und Umwelteinflüsse $\mathrm{zu}$, so dass keine gesicherten genetischen Unterschiede nachgewiesen werden können. Obwohl nicht gesichert, wird das Phänomen deutlich, dass die heterozygoten Genotypvarianten $\kappa-\mathrm{CN}^{\mathrm{AB}}$ und $\beta-L G^{\mathrm{AB}}$ in beiden Stichproben SR und RP bei allen untersuchten Nutzungsmerkmalen nahezu überlegen sind.

Tabelle 6

Nutzungsdauer bei Schwarzbunten nach $\kappa$-Casein und $\beta$-Lactoglobulin-Genotypvarianten (Production performance in Black-and White breed due to milk k-casein and $\beta$-lactoglobulin genotyps)

\begin{tabular}{|c|c|c|c|c|c|}
\hline Genotyp & Symbol & $\begin{array}{c}\text { Erstkalbealter in } \\
\text { Tagen }\end{array}$ & $\begin{array}{l}\text { Zwischenkalbezeit } \\
\text { in Tagen }\end{array}$ & $\begin{array}{l}\text { Lebensdauer in } \\
\text { Monaten }\end{array}$ & $\begin{array}{c}\text { Laktationen im } \\
\text { Leben }\end{array}$ \\
\hline \multicolumn{6}{|c|}{$\mathrm{k}$-Casein } \\
\hline AA & $\bar{x}$ & $829^{b}$ & 429 & 83,0 & 4,54 \\
\hline & $\hat{\mathrm{s}}$ & 59 & 86 & 22,2 & 1,90 \\
\hline $\mathrm{AB}$ & $\frac{7}{x}$ & $807^{\star}$ & 384 & 86,6 & 4,85 \\
\hline & $\hat{s}$ & 66 & 67 & 22,5 & 1,88 \\
\hline BB & $\bar{x}$ & 831 & 414 & 95,6 & 5,50 \\
\hline & $\mathrm{s}$ & 68 & 60 & 21,6 & 1,85 \\
\hline \multicolumn{6}{|c|}{$\beta$-Lactoglobulin } \\
\hline $\mathrm{AA}$ & $\mathrm{x}$ & 821 & 419 & 80,9 & 4,41 \\
\hline & $\hat{s}$ & 58 & 54 & 16,4 & 1,69 \\
\hline $\mathrm{AB}$ & $\bar{x}$ & $812^{\prime \prime}$ & 409 & 88,7 & 5,07 \\
\hline \multirow{3}{*}{ BB } & $\hat{s}$ & 68 & 88 & 25,4 & 2,10 \\
\hline & $\bar{x}$ & $839^{6}$ & 418 & 82,5 & 4,36 \\
\hline & $\frac{\alpha}{s}$ & 50 & 79 & 21,0 & 1,71 \\
\hline
\end{tabular}

.6. Signifikanz $\mathrm{P}=5 \%$ 
Tabelle 7

Nutzungsdauer beim Polnischen Rotvieh nach $k$-Casein und $\beta$-Lactoglobulin-Genotypvarianten (Production performance in Polish Red breed due to milk k-casein and $\beta$-lactoglobulin genotyps)

\begin{tabular}{|c|c|c|c|c|c|}
\hline Genotyp & Symbol & $\begin{array}{c}\text { Erstkalbealter in } \\
\text { Tagen }\end{array}$ & $\begin{array}{c}\text { Zwischenkalbezeit } \\
\text { in Tagen }\end{array}$ & $\begin{array}{c}\text { Lebensdauer in } \\
\text { Monaten }\end{array}$ & $\begin{array}{l}\text { Laktationen } \\
\text { im Leben }\end{array}$ \\
\hline \multicolumn{6}{|c|}{ K-Casein } \\
\hline AA & $x$ & 836 & 375 & 59,2 & 3,05 \\
\hline & s & 64 & 28 & 24,6 & 1,99 \\
\hline $\mathrm{AB}$ & $\bar{x}$ & $830^{\circ}$ & 381 & 62,7 & 3,38 \\
\hline & $\hat{s}$ & 65 & 36 & 20,8 & 1,70 \\
\hline BB & $\bar{x}$ & $880^{b}$ & 395 & 64,2 & 3,20 \\
\hline & $\mathrm{s}$ & 83 & 35 & 17,2 & 1,62 \\
\hline & & & $\beta$-Lactoglobulin & & \\
\hline$\overline{A A}$ & $x$ & 823 & 359 & 47,9 & 2,33 \\
\hline \multirow{3}{*}{$A B$} & $\begin{array}{l}\lambda \\
s\end{array}$ & 77 & 28 & 9,9 & 0,52 \\
\hline & $\bar{x}$ & 854 & 385 & 59,8 & 2,89 \\
\hline & s & 71 & 34 & 22,6 & 1,81 \\
\hline \multirow[t]{2}{*}{ BB } & $\bar{x}$ & 833 & 383 & 63,0 & 3,38 \\
\hline & s & 65 & 34 & 22,4 & 1,80 \\
\hline
\end{tabular}

\section{Diskussion}

Die Milchproteinpolymorphismen wurden nach den Genotypvarianten des $\kappa$-Caseins ( $\kappa-C N)$ und des $\beta$-Lactoglobulins ( $\beta$-LG) an 362 Kühen mit ihren Jungkuhleistungen in der ersten Laktation, dem Erstkalbealter, der Zwischenkalbezeit, der Lebensdauer und der Anzahl Laktationen untersucht. Die beiden einbezogenen Teilpopulationen Polnische Schwarzbunte (SR) und Polnisches Rotvieh (RP) unterscheiden sich in ihrem Leistungsniveau erheblich mit etwa:

$5300 \mathrm{~kg}$ Milch, $210 \mathrm{~kg}$ Fett und $170 \mathrm{~kg}$ Protein bei 4,0\% Fett und 3,2\% Protein für SR und $2800 \mathrm{~kg}$ Milch, $120 \mathrm{~kg}$ Fett und $90 \mathrm{~kg}$ Protein bei 4,3\% Fett und 3,2\% Protein für RP.

Bei dem veränderten Leistungsniveau der Rassen bzw. Teilpopulationen sind veränderte Allelfrequenzen zu beobachten. Der Anteil der Allelfrequenz des $\kappa-\mathrm{CN}^{\mathrm{AA}}$ steigt von RP zu SR um $10 \%$ von 68 auf $78 \%$ ebenso wie der Anteil der Allelfrequenz des $\beta-\mathrm{LG}$ steigt von RP zu SR um $28 \%$ von 22 auf $50 \%$. Die veränderte Relation zwischen Allel A und B beim K-Casein von etwa 70:30 bei RP auf 80:20 bei SR sowie beim $\beta$-Lactoglobulin von 22:74 bei RP auf etwa 50:50 bei SR ist mit einem Leistungsanstieg auch in anderen Schwarzbunt-Populationen zu finden (ERHARDT, 1996).

In den Stichproben beider Rassen haben Kühe mit dem к-CN-Genotyp BB ungesichert den höchsten prozentualen Proteingehalt in der Milch und diese haben die höchste Proteinleistung erreicht. Ähnliche Ergebnisse erreichten PANICKE et al. (1997) für den Proteingehalt in der Milch bei gleichzeitiger Überlegenheit des. Fettgehaltes dieser $\kappa-\mathrm{CN}$-Genotypvariante BB im Unterschied zu diesen vorliegenden Untersuchungen. Ebenso konnte die Überlegenheit der heterozygoten Genotypvarianten im Fettertrag bei SOWINSKI (1993) nicht wiederholt werden. Es ist zu erwarten, dass die Zusammenhänge zwischen verschiedenen Merkmalen der Milchleistung bei beiden Rassen und den Milchproteinvarianten dem Einfluss der Genotyp-Umwelt Interak- 
tionen unterliegen, wie sie z.B. auch schon bei JANICKI (1974) oder KRIVENCOW et al. (1977) im Zusammenhang zwischen dem $\beta$-Lactoglobulin und dem prozentualen Fettgehalt in Abhängigkeit vom Leistungsniveau der Herde beschrieben wurden. Aufgrund der engen Kopplung zwischen den Caseingenorten, treten einzelne Allele an zwei Caseingenorten häufiger miteinander auf, als dies unter der Annahme eines Kopplungsgleichgewichtes erwartet wird. Dies bedeutet, dass die einzelnen Genotypen nicht unabhängig von einander betrachtet werden können. Nach ERHARDT (2000) ist deshalb die Verwendung von zusammengesetzten Genotypen eine Möglichkeit, um den Effekt von Caseingenotypen auf verschiedene Merkmale zu schätzen. Dies erfordert einen höheren Versuchsumfang.

Für die Ergebnisse der Nutzungsdauer der untersuchten Stichproben aus beiden Teilpopulationen konnte kein Zusammenhang $\mathrm{zu}$ den $\mathrm{k}-\mathrm{CN}$ - und $\beta-\mathrm{LG}-\mathrm{Genotypen}$ gesichert werden. Es gibt nur einen gesicherten Zusammenhang zum Erstkalbealter für die Genotypen $\kappa-C N^{A A}>\kappa-C N^{A B}$ und für den Genotyp $\beta-L G^{B B}>\beta-L G^{A B}$ bei SR sowie für $\kappa-\mathrm{CN}^{\mathrm{BB}}>\mathrm{K}-\mathrm{CN}^{\mathrm{AB}}$ beim RP entsprechend mit 22,27 und 50 Tagen. Die Kühe aus beiden Rassen mit verschiedenen Milchproteinvarianten weisen gesicherte Unterschiede im Erstkalbealter um fast einen Monat aus:

$\begin{array}{lcccccc}\text { Beim } & \kappa-\mathrm{CN}^{\mathrm{AA}} & \kappa-\mathrm{CN}^{\mathrm{BB}} & \kappa-\mathrm{CN}^{\wedge \mathrm{B}} & \text { oder } \beta-\mathrm{LG}^{\wedge A} & \beta-\mathrm{LG}^{\mathrm{BB}} & \beta-\mathrm{LG}^{\mathrm{AB}} \\ \text { bei SR } & 27,2 & (27,2) & 26,4 & (26,9) & 27,5 & 26,6 \\ \text { bei RP } & (26,9) & 28,9 & 27,1 & & & \end{array}$

Im weiteren Lebensabschnitt bis zum Ausscheiden aus der Produktion gewinnen Umwelteinflüsse zunehmend kumulativ an Einfluss auf die untersuchten Parameter Zwischenkalbezeit und Lebensdauer.

Aus den Tabellen 6 und 7 wird die Tendenz deutlich, wenn dies auch nicht gesichert werden konnte, dass die heterozygoten Genotypvarianten $\mathrm{K}-\mathrm{CN}{ }^{\mathrm{AB}}$ und $\beta-\mathrm{LG}^{\mathrm{AB}}$ in beiden Stichproben SR und RP bei allen untersuchten Nutzungsmerkmalen nahezu uberlegen sind. Diese Tendenzen sind an größeren Kuhpopulationen in Clustern mit aggregiertem Genotypen bei größerem Versuchsumfang zielgerichtet weiter zu verfolgen,

\section{Literatur}

AALLTONEN, M.L.; ANTILA, V.: Milk renneting properties nd the genetic variants of proteins. Milchwissenschaft 42 (1987), 490-492

ALEANDRI, R.; BUTTAZZONI, L.; PAGGI, U.; DAVOLI, R.; NANNI KOSTA, L.; RUSSO, V.:

Effect of bovine milk proteinpolymorphism at two loci on cheese producing ability. IDF Milk Protein Polymorphism II Seminar, 24.-27.02.1997, Palmerston North, Neuseeland

ERHARDT, G.:

$\kappa$-Casein in der Rindermilch - Nachweis eines weiteren. Allels ( $\kappa$-casein ${ }^{E}$ ) in verschiedenen ERHARDT,G.:

Allele frequencies of milk proteins in German cattle breeds and demonstration of $\alpha_{\$ 2}$-casein variani by isoelectric focussing. Arch. Tierz., Dummerstorf 36 (1993) 2, 145-152

ERHARDT, G. ; KRICK-SALECK, H.; JUSZCZAK,J.; PANICKE, L.

Zusammenhänge zwischen dem Vorkommen von genetisch bedingten Varianten in den Milchproteinen und der Verwandtschaft von Rinderrassen. Schriftenreihe des FBN Dummerstorf, Heft 13, 1996, 3-9 
ERHARDT, G.:

Assoziation von DNA-Polymorphismen der Milchproteingene und des Wachstumshormongens mit quantitativen und qualitativen Merkmalen. Schriftenreihe des FBN Dummerstorf, Heft 13, 2000, 89-94

FELENCZAK, A.; ORMIAN, M,; SZAREK, J.:

Interrelationship between milk protein content and occurrence of genetically determine protein fractions. Genetica Polonica, 24 (1983), 355-362

FERRETTI, L.; LEONE, P.; SGARAMELLA, V; Long range restriction analysis of the bovine casein genes. Research 18 (1990), 6829-6833

GRAML, R.; BUCHBERGER, J.; PIRCHNER, F.: Zuchtung auf Kăsereitauglichkeit der Milch? Zuchtungskunde, Stutigart 60 (1988), 11-23

JANICKI, C.:

Próba wykorzystania beta-laktoglobulin w selekcji krów mlecznych rasy nizinnej czmo-białej. Rocz. AR, Pozn. 2 (1974), 14-19

KRIVENCOW, J.M; SMETANINA, G.K.; EM'LJANOW, A.S.:

Belkovomolocnost cerno-pestrogo skota i ee vzajmosvja'z s tipami $\beta$-laktoglobulinow. Doklady VASCHIL. (1977), 30-31

MARIANI, P,; LEONI, M.:

Il tempo di coagulazione del latte in rapporto alle vario genetiche delle caseine $\beta$ e $\kappa$. Ann. Fac, Med. Vet, Univ. Parma. 5 (1985), 185-19S

MARIANI, P.; PECORARI, M.P.:

Fattori genetici attitudine alla caseficazione e resollatte in formaggio. Sci. Tecn. Latt.-cas. 38 (1987), 286-326

MARZIALI, S.; NG-KWAI-HANG, K.F.:

Effects of milk composition and genetic polimorphisms on coagulation properties of milk. J. Dairy Sci. 69 (1986), 1793-1798

NG-KWAI-HANG, K.F; HAYES, J.F.; MOXLEY, J.E.; MONARDES, H.G.:

Relationships between milk protein polymorphisms and major milk constituants in HolsteinFriesian cows. J, Dairy Sci. 69 (1986), 22-26

PANICKE, L.; FREYER, G.; ERHARDT, G.; SCHLETTWEIN, K.: Milchproteingenotypen und ihr Einflu $\beta$ auf die Milchleistungsmerkmale. Arch. Tierz, Dummerstorf 39 (1996), 3-16

PANICKE, L.; FREYER, G.; ERHARDT, G.:

Effects of milk protein genotypes on milk produktion traits. EAAP Vienna G1.14, (1997)

PANICKE,L.; FREYER;G.; ERHARDT,G.;

Effekte von Milchproteinvarianten auf die Fruchtbarkeitseigenschaften beim Schwarzbunten Milchrind. Arch. Tierz. Dummerstorf 41 (1998) 5, 447-454

SCHAAR, J:

Variation in milk protein composition. Swedish University of Agricultural Sciences. Rapport 71 Uppsala (1986)

SCHULTE-COERNE, H.; PABST, K.:

Relevance of casein genotypes for the genetic variance of cheese making properties and heat stability. EAAP Berlin C2.4, (1991)

SEIBERT, B.; ERHARDT, G.; SENFT, B.:

Procedure for simultaneous phenotyping of genetic variants in cow's milk by isoelectric focusing. Anim. Blood Grps. and Biochem. Genet. 16 (I985), 183-19I

SOWINSKI, G.:

Związek genetycznych wariantów $\beta$-laktoglobuliny, $\alpha_{s 1}-, \beta$ - oraz $\kappa$-kazein z wydajnością, składem chemicznym i wskaźnikami technilogicznej przydatności mleka krów rasy nizinnej czamo-białej. Acta Acad. Agricult. Tech.Olst, Zootechnika 38 (1993), 2-38

TRAKOVICKA, A.; KUBEK, A,; GAVALIER, M.; ZITNY, J::

Genotypes of $\mathrm{K}-\mathrm{Cn}$ and milk produktion of cows selected according to type and body conformation. EAAP Viena GI.11 (1997)

WALAWSKI, K.; CZARNIK, U,; ZABOLEWICZ, T.:

Związek pomiędzy polimorfizmem betalaktoglobuliny (BLG) i zróźnicowaniem wskaźników diagnostycznych charakteryzujących podkliniczne stany mastitis u krów rasy czamo-białej. Rocz. Nauk. Zoot. 24 (1997), 9-22 
ŻUK, B.; SZYSZKOWSKI, L.; FILISTOWICZ, A.:

Poprawki na wiek i sezon ocielenia dla korygowania cech mleczności krów rasy czerwono-białej 1 czarno-bialej w Polsce poludniowo-zachodniej. Roczn. Nauk Roln. S.B. 100 (1980), 53-66

lingegangen: 03.02.2000

Akzeptiert: 02.04 .2001

Anschriften der Vetfasser

Prof. Dr. Dr, habil. JERZY JUSZCZAK, Prof. Dr, habil. RYSZRD ZIEMIŃSKI,

Dr. MARIAN KUCZAJ

Landwirtschaftliche Universitat Wroclaw,

Lehrstuhl für Rinderzucht und Milchproduktion

Kozuchowska 5-b

51-631 Wroclaw

Polen

Prof. Dr. GEORG ERHARDT

Justus-Liebig-Universität Giessen

Institut für Tierzucht und Haustiergenetik

Ludwigstr. 21B

D- 35390 Giessen

Prof. Dr. habil. LOTHAR PANICKE

Forschungsinstitut für die Biologie Landwirtschaftlicher Nutztiere

Wilhelm-Stahl-Allee 2

D- 18196 Dummerstorf 
Arch. Tierz., Dummerstorf 44 (2001) 3, 250

\title{
Buchbesprechung
}

\author{
Seltene Haus- und Nutztierrassen
}

MARTIN HALLER

176 Seiten, 130 farbige Abbildungen, Leopold Stocker Verlag, Graz, Stuttgart, 2000

ISBN 3-7020-0893-4, DM 39,90; ชS 291,00; sFr 37,00

Die sich in Jahrhunderten entwickelte Vielfalt unserer Haus- und Nutztierrassen hat sich in den letzten Jahrzehnten erheblich eingeschrånkt. Okonomische Zwănge, Technisierung, Leistungsanforderungen und weitere Grunde haben zu Rassenverschmelzungen oder den Verlust von Rassen gefuhrt. Bei anderen seltenen Rassen gefahhrdet der Bestandsriuckgang den für die Reproduktion notwendigen Tierbestand um diese Population zu erhaltenen. Als Folge dieser Entwicklung droht letztlich ein unersetzlicher Verlust spezieller, genetisch fundierter Eigenschaften vor allem im allgemeinen Leistungsbereich. Für viele dieser Rassen erscheint dieses Buch gerade zur rechten Zeit um die Aufmerksamkeit auf diese Tiere zu lenken. Diese Rassen zeichnen sich durch ihre relative Anspruchslosigkeit aus und prägen besondere Landschaftsbilder. Sie stellen ein unverzichtbares Kulturgut dar, welches wie anderes, erhalten und gepflegt werden muss. Passionierte Zlichter, Tierzuchtwissenschaftler und Tierfreunde haben seit Jahren nicht nur auf diesen besorgniserregenden Umstand hingewiesen, sondern die Bemuhungen unterstlitzı noch verfügbare Tierbestände der gefahrdeten Rassen zu erfassen und Möglichkeiten ihrer Bewahrung aufzuzeigen. Es ist ein bleibendes Verdienst des Autors, mit vorliegendem Buch, dieses Anliegen zu unterstützen. Das Buch wird mit dazu beitragen uber solche wichtigen seltenen Rassen zu informieren, die vor allem im deutschsprachigen Raum heimisch waren und sind.

Die Biographien der 115 vorgestellten Rassen umfassen bei Pferd und Esel 20, beim Rind 24, beim Schwein 8 , beim Schaf 16, bei der Ziege 12 und beim Geflugel 35 Populationen mit ihrer Vorstellung in Wort und Bild. Im ersten Kapitel werden u.a. wichtige Organisationen des Artenschutzes in Deutschland, der Schweiz und Österreich mit ihren Zielen, Organisationsformen und ihrer Arbeit vorgestellt. Das nunmehr seit 30 Jahren aktive und vielfaltige Engagement der "Deutschen Gesellschaft filr Zuchtungskunde" zur Erhaltung der genetischen Vielfalt bei Nutztierrassen bleibt hier leider unerwahnt. Den Hauptirhalt des Buches bilden die sehr sorgfaltig recherchierten, nach Tierarten geordneten, mit schonen Farbbildern anschaulich unterstutzten, instruktiven Rassebeschreibungen. Fur jede Rasse kann der Leser Informationen aber ihre Herkunf, die Rassengeschichte, die Verbreitung bis in die Gegenwart, die Standorteignung und die besonderen Eigenschaften dieser Population entnehmen.

Dieses vom Verlag sehr gut ausgestattete Buch wird Landwirten, Tierzuchtern, Tierfreunden und allen an der Haus- und Nutztierhaltung interessierten Lesern ebenso nutzen, wie es fur Lehrer, in der Beratung Tatige, Studenten tangierender Fachdisziplinen und Biologen wichtige Informationen bereithalt. Die gelungene Kombination von Bild- und Textinformation empfiehlt dieses Buch aber auch Tierfreunden, die sich anhand schöner Bilder an der Vielfalt unserer Haustierpopulationen erfreuen mðchten. 